\title{
25 Research Soure \\ The variation of pelvis in unilateral Crowe type IV developmental dysplasia of the hip
}

\author{
Yinqiao Du \\ Chinese PLA General Hospital \\ Jingyang Sun \\ Chinese PLA General Hospital \\ Haiyang Ma \\ Chinese PLA General Hospital \\ Junmin Shen \\ Chinese PLA General Hospital \\ Ming Ni \\ Chinese PLA General Hospital \\ Yonggang Zhou ( $\nabla$ ygzhou301@163.com ) \\ 301 hospital https://orcid.org/0000-0002-7304-4691
}

\section{Research article}

Keywords: developmental dysplasia of the hip, pelvic landmarks, preoperative planning, total hip arthroplasty

Posted Date: February 17th, 2020

DOl: https://doi.org/10.21203/rs.2.23714/v1

License: (c) (1) This work is licensed under a Creative Commons Attribution 4.0 International License. Read Full License

Version of Record: A version of this preprint was published at Orthopaedic Surgery on February 22nd, 2021. See the published version at https://doi.org/10.1111/os.12903. 


\section{Abstract}

\section{Background}

The whole pelvic morphology is very common in developmental dysplasia of the hip (DDH). The abnormalities may influence the pelvic landmarks, and then misguide the surgeon's preoperative plan. The purpose of this study was to investigate the variation of pelvis in unilateral Crowe type IV DDH and analyze the reliability of pelvic landmarks.

\section{Methods}

We received preoperative anteroposterior pelvic radiographs examined for 89 adult patients with unilateral Crowe type IV DDH at our institution between September 2008 and May 2019. 48 patients without a false acetabulum was type IVA and 41 with a false acetabulum was type IVB. The heights of the ilium, acetabulum and ischium areas in affected and unaffected sides were measured, the discrepancies of bilateral iliac crest, inferior sacroiliac articulation, teardrop and ischial tuberosity on the bisector of the pelvis were also measured.

\section{Results}

The mean heights of the ilium, acetabulum, ischium areas in the affected side were $74.4 \mathrm{~mm}, 88.6 \mathrm{~mm}$ and $37.0 \mathrm{~mm}$, respectively, in type IVA group and $77.7 \mathrm{~mm}, 83.5 \mathrm{~mm}$ and $37.8 \mathrm{~mm}$, respectively, in type IVB group. The heights in the unaffected side were $82.1 \mathrm{~mm}, 84.6 \mathrm{~mm}$ and $43.8 \mathrm{~mm}$, respectively, in type IVA group and $84.6 \mathrm{~mm}, 82.0 \mathrm{~mm}$ and $44.0 \mathrm{~mm}$, respectively, in type IVB group. The discrepancies of bilateral iliac crest, inferior sacroiliac articulation, teardrop and ischial tuberosity on the line of the bisector of the pelvis were $6.7 \mathrm{~mm}, 4.4 \mathrm{~mm}, 2.7 \mathrm{~mm}$ and $6.1 \mathrm{~mm}$, respectively.

\section{Conclusions}

The pelvic asymmetry was a common occurrence in adults unilateral Crowe type IV DDH. Furthermore, it should be reliable to use teardrop as pelvic landmark to balance leg length discrepancy in preoperative planning.

\section{Background}

Unilateral Crowe type IV developmental dysplasia of the hip (DDH) consists of several morphologic anomalies both femoral and pelvic sides. Many studies concerned the shape of the proximal femur and the acetabulum, including a narrower and stovepipe shaped medullary canal, a small diaphyseal diameter, excessive femoral anteversion, high neck-shaft angles and a shallow acetabulum [1-5]. However, few studies have explored the variation of the whole pelvic morphology in Crowe type IV DDH. These may include the pelvic asymmetry and rotation[6, 7]. Pelvic radiograph is widely used for measuring leg length discrepancy, and preoperative and intraoperative evaluations using the proper pelvic landmarks, including iliac crest, inferior sacroiliac articulation, teardrop and ischial tuberosity [8-13]. All of 
these abnormalities may influence the pelvic landmarks, and then misguide the surgeon's preoperative plan. We conducted a study to radiologically examine the effect of Crowe type IV on pelvic development in adult with unilateral DDH. In this study we investigated how Crowe type IV DDH affected development of the pelvis, and analyzed the reliability of pelvic landmarks to balance leg length discrepancy for unilateral DDH patients in preoperative planning.

\section{Methods}

We received preoperative anteroposterior (AP) pelvic radiographs examined for 89 patients with unilateral Crowe type IV DDH, who underwent total hip arthroplasty (THA) at our institution between September 2008 and May 2019. Since non-operative or operative treatment can alter the pelvic anatomy, patients with a history of prior treatment were excluded. The present research also did not involve the DDH for infection or trauma. All of the 89 patients were divided into two groups according to whether there is a false acetabulum [14]. 48 patients without a false acetabulum was type IVA and 41 with a false acetabulum was type IVB. Patient demographic characteristics of the two groups were showed in the Table 1. The two groups were similar in terms of gender, age, height, weight and body mass index. Our hospital's institution review board approved the study.

A standardized radiographic technique was performed for all received AP pelvic radiographs. All radiographs were performed in the supine position. A film focus distance of $1.2 \mathrm{~m}$ was used with the beam between the pubic symphysis and a line connecting the anterior superior iliac spine with the pelvis in neutral rotation $[15,16]$. The longitudinal rotation of the pelvis was verified as correct when the tip of coccyx was in line with pubic symphysis.

All the radiographs were viewed and measured on a picture archiving and communication system (PACS, UniWeb Viewer, version 4.0, EBM technologies). Both sides of the pelvis were separated into three areas by vertical lines drawn over the bisector of the pelvis (a line connecting middle point of the lower edge of fifth lumbar vertebrae with pubic symphysis) from ischial tuberosity, from teardrop, from inferior sacroiliac articulation, from iliac crest. The area containing the iliac wing was ilium area, the acetabulum as acetabulum area, and the ischium as ischium area [8]. The height of each area was calculated as a proportion of the height of the entire pelvis on the same side of the body to nullify the confounding effects of patient body size.

Four measurements were made bilaterally on AP pelvic radiographs of each patient (Table 2 and Fig. 1): (1) height of ilium area, (2) height of acetabulum area, (3) height of ischium area, (4) width of ilium. Another four measurements were made on radiographs: the discrepancies of bilateral iliac crest, inferior sacroiliac articulation, teardrop and ischial tuberosity on the line of the bisector of the pelvis (Table 2 and Fig. 1). Two independence investigators (YQD and JYS), who were familiar with digital measurement, performed the radiographic measurements. All of the measurements were repeated four weeks later to assess the intra-observer reliability. Inter- and intraobserver reliabilities were tested for each parameter using the intraclass correlation coefficient (ICC) (Table 2). 
Categorical data were compared using a chi-squared test. The paired samples $t$ test or Wilcoxon test was used to compare continuous data between the affected and unaffected sides. The independent-samples $t$ test or Mann-Whitney $\mathrm{U}$ test was used to compare continuous data between the type IVA and type IVB groups. The Friedman's two-way ANOVA was used to compared continuous data between the discrepancy of bilateral iliac crest, inferior sacroiliac articulation, teardrop and ischial tuberosity on the line of the bisector of the pelvis. All tests were performed using SPSS version 26 (IBM Corp., Armonk, NY). Continuous data were given as the mean and standard deviation. A $p$-value $<0.05$ was considered significant in all analysis.

\section{Results}

In Crowe type IVA group, the mean heights of the ilium, acetabulum, ischium areas in the affected side were $74.4 \mathrm{~mm}, 88.6 \mathrm{~mm}$ and $37.0 \mathrm{~mm}$, respectively, the heights in the unaffected side were $82.1 \mathrm{~mm}$, $84.6 \mathrm{~mm}$ and $43.8 \mathrm{~mm}$, respectively (ilium area, $\mathrm{P}<0.001$; acetabulum area, $\mathrm{P}<0.001$; ischium area, $\mathrm{P}<$ 0.001 ). The mean height of the entire pelvis both affected and unaffected sides were $200.0 \mathrm{~mm}$ and $210.5 \mathrm{~mm}$, respectively $(P<0.001)$. The mean width of ilium both affected and unaffected sides were $87.6 \mathrm{~mm}$ and $105.0 \mathrm{~mm}$, respectively $(P<0.001)$. In Crowe type IVB group, the mean height of the three areas in the affected side were $77.7 \mathrm{~mm}, 83.5 \mathrm{~mm}$ and $37.8 \mathrm{~mm}$, respectively, the height in the unaffected side were $84.6 \mathrm{~mm}, 82.0 \mathrm{~mm}$ and $44.0 \mathrm{~mm}$, respectively (ilium area, $\mathrm{P}<0.001$; acetabulum area, $\mathrm{P}=0.032$; ischium area, $\mathrm{P}<0.001)$. The mean height of the entire pelvis both affected and unaffected sides were $199.1 \mathrm{~mm}$ and $210.6 \mathrm{~mm}$, respectively $(P<0.001)$. The mean width of ilium both affected and unaffected sides were $98.6 \mathrm{~mm}$ and $100.2 \mathrm{~mm}$, respectively $(P=0.387)$. The height of the ilium area in affected side of Crowe type IVB group was higher than that in affected side of Crowe type IVA group, but there was no significant difference $(P=0.110)$. However, the height of the acetabulum area in affected side of Crowe type IVB group was significantly lower than that in affected side of Crowe type IVA group $(P=0.018)$. There was no significant difference in the height of ischium area of the affected side between the type IVA and IVB groups $(P=0.434)$. The height of entire pelvis of the affected side between the type IVA and IVB groups was also no significant difference $(P=0.778)$. However, the width of ilium in affected side of Crowe type IVB group was significantly greater than that in affected side of Crowe type IVA group $(\mathrm{P}<$ $0.001)$.

The ratios of the ilium, acetabulum, ischium areas in affected side of Crowe type IVA group were 0.37, 0.44 and 0.19 , respectively, and the ratios in unaffected side were $0.39,0.40$ and 0.21 , respectively (ilium area, $\mathrm{P}<0.001$; acetabulum area, $\mathrm{P}<0.001$; ischium area, $\mathrm{P}<0.001$ ). The ratios of the ilium, acetabulum, ischium areas in affected side of Crowe type IVB group were $0.39,0.42$ and 0.19 , respectively, and the ratios in unaffected side were $0.40,0.39$ and 0.21 , respectively (ilium area, $P<0.001$; acetabulum area, $P$ $<0.001$; ischium area, $P<0.001)$. The ratio of the ilium area in affected side of Crowe type IVB group was significantly greater than that in affected side of Crowe type IVA group $(P=0.005)$. The ratio of the acetabulum area in affected side of Crowe type IVB group was significantly lower than that in affected side of Crowe type IVA group $(P=0.010)$. The difference of the ratio of the ischium area of affected side in Crowe type IVB and IVB groups was no significant $(P=0.281)$. 
The discrepancies of bilateral iliac crest, inferior sacroiliac articulation, teardrop and ischial tuberosity on the line of the bisector of the pelvis were $6.7 \mathrm{~mm}(0$ to 19.6$), 4.4 \mathrm{~mm}(0$ to 16.4$), 2.7 \mathrm{~mm}$ (0 to 17.5) and $6.1 \mathrm{~mm}(0$ to 13.7), respectively (teardrop versus iliac crest, $P<0.001$; teardrop versus inferior sacroiliac articulation, $P<0.001$; teardrop versus ischial tuberosity, $P<0.001$ ) (Fig. 2).

\section{Discussion}

Although the morphologic features around the hip in Crowe type IV DDH have been well described [1, 2, 5, 17], the morphologic variation of the entire pelvic in unilateral Crowe type IV DDH are not well characterized and the pelvic landmarks association with morphologic features of the pelvis are also unclear. We focused on the development of pelvis and the reliability of pelvic landmarks in the patients with unilateral Crowe type IV DDH on AP pelvic radiographs.

We acknowledge that our study has a number of limitations. First, this is a retrospective study. Second, we selected the patients with unilateral Crowe type IV DDH, but there are no healthy patients to compare. Although we compared the variation of pelvic both affected and unaffected sides, it is unclear that the difference between the unaffected side and the healthy pelvis. Third, we were unable to measure the actual parameter of the pelvis on 2D AP pelvic radiographs. CT could nullify the confounding effects of the pelvic rotation and provide precise measurements to determine the variation of pelvic in both coronal and sagittal planes. However, all the parameters are vertical distance excepted the width of ilium, the ratios of three areas in entire pelvis are calculated to ensure the results were reliable. The AP radiographs are the most frequently the first, and sometimes the only, imaging studies performed on patients seeking treatment for hip disease because of that is faster and more universally accessible.

The structural abnormalities exist throughout the pelvis in $\mathrm{DDH}$, and the morphologic abnormalities of the acetabulum are not solely resulted in local dysplasia around the hip, but are influenced by the morphologic features of the entire pelvis [18]. A study of eight-three cases of unilateral late-diagnosed (older than 4 months of age) DDH showed the pelvic asymmetry was evident in DDH, possibly secondary to growth of pelvic disturbance in the triradiate cartilage [6]. The pelvic asymmetry was also a common occurrence in adults DDH according to our study. Fujii et al. found the internal rotation from the iliac crest through ischial tuberosity in DDH group was significantly greater than that in normal group by using the CT measurement [18]. Bilgen et al. divided unilateral pelvic dysplasia into three areas with the following four lines in Crowe type II, III, and IV DDH: connecting the bilateral iliac crest vertex, connecting the bilateral inferior sacroiliac articulation, connecting the bilateral teardrop, and connecting the bilateral ischial tuberosity. They showed the height of acetabular area of affected side was significantly larger than that of the contralateral side, which is thought to originate from the effect created in the distal acetabulum, with an increased reactionary effect on the iliopsoas capsule and upward tension of dislocation femoral head on the capsule [8]. In our study, we got the same results in the height of acetabular area in Crowe type IV DDH. However, compared with the bilateral discrepancy of the height of acetabular area in Crowe type IVA group, the bilateral discrepancy in Crowe type IVB group was narrower. The bilateral discrepancy of the height of ilium area in Crowe type IVB group was also narrower than that 
in Crowe type IVA group. The width of ilium of affected side was closer to the unaffected side in Crowe type IVB group. In type IVB DDH, the femoral heads articulate with the iliac wing to form a pseudarthrosis, the false acetabulum may be an important factor to facilitate the development of the ilium area. The presence and absence of a false acetabulum in Crowe type IV DDH are associated with different loading patterns and different soft tissue condition, which may result in different morphologic features of the pelvis and the femur[2]. However, the false acetabulum has no effect on the development of ischium area. According to our results, the height of the ischium area in the affected side was significantly lower than that in the unaffected side, which may due to the absence of the growth-stimulating of the centralized pressure from femoral head to result in abnormal development of the ischium and the pubis in the axial plane after the femoral head dislocated.

Pelvic radiograph is widely used for measuring leg length discrepancy, and preoperative and intraoperative evaluations using the proper pelvic landmarks, including iliac crest, inferior sacroiliac articulation, teardrop and ischial tuberosity. The pelvic asymmetry and rotation may influence the pelvic landmarks in Crowe type IV DDH. Li et al. didn't recommend the teardrop was used as landmark to balance leg length discrepancy for unilateral DDH patients in preoperative planning and THA by measuring the vertical distance from the teardrop to the line across the bottom edges of bilateral ischial tuberosity [19]. However, the variation of ischial tubercle was not considered in the measurement. The using a line crossing the healthy hip's teardrop and parallel to a line jointing the inferior sacroiliac articulation is useful for calculating leg length discrepancy for unilateral Crowe type IV DDH patients in preoperative planning due to the distance between the above parallel line crossing teardrop and the teardrop in the affected side was approximately $10.04 \mathrm{~mm}$ according to the study by Bilgen et al. [8]. Meermans et al. demonstrated that the teardrop was more reliable than the bi-ischial line, particularly in patients with leg length discrepancy [20]. In our study, we selected the line of the bisector of the pelvis as the base line to analyze the variation of the pelvic landmarks. The discrepancies of bilateral teardrop on the line of the bisector of the pelvis was significantly smaller than that of iliac crest, inferior sacroiliac articulation and ischial tuberosity. Therefore, we suggest that it should be reliable to use teardrop as pelvic landmark to balance leg length discrepancy for unilateral Crowe IV DDH patients in preoperative planning and THA. And the teardrop has a separate anatomic structure, and thus there will be no effect on the measurement od pelvic rotation in the vertical position [21].

Appearance of a teardrop figure in AP pelvic radiographs is directly related to the growth and development of the acetabulum [22]. Therefore, the anatomic abnormality of teardrop is associated with DDH in adult patients. The teardrop may be seen between 6 and 24 months of age in the literature [2325]. Albinana et al. stated that there was progressive widening of the teardrop in the affected side, the increase was first in the superior width at 2 years and then the inferior width at 10 years old of the children [23]. Erkula et al. found the V-shaped teardrop was directly related to hip dysplasia in children patients [22]. According to our observation, the $V$-shape teardrop was also a common occurrence in adults DDH. and should be identified by surgeons. 


\section{Conclusions}

The pelvic asymmetry was a common occurrence in adults unilateral Crowe type IV DDH. The false acetabulum may facilitate the development of the ilium and acetabular areas. Furthermore, it should be reliable to use teardrop as pelvic landmark to balance leg length discrepancy for unilateral Crowe IV DDH patients in preoperative planning and THA.

\section{Abbreviations}

DDH: Developmental dislocation of the hip; AP: anteroposterior; THA: Total hip arthroplasty; BMI: body mass index; COR: center of rotation.

\section{Declarations}

\section{Ethics approval and consent to participate}

The Ethics Committee of our hospital, General Hospital of Chinese People's Liberation Army, approved the study protocol. All the study participants provided written informed consent for the study.

\section{Consent for publication}

Written informed consent was obtained from all patients for publication of this study and any accompanying images.

\section{Availability of date and materials}

The data will be made available from the authors upon reasonable request.

\section{Competing interests}

The authors declare that they have no competing interests.

\section{Funding}

This study was funded by National Key Research and Development Program of China (N.0.2017YFB1104104). The funding body played a role in data collection and analysis.

\section{Authors' contributions}

YGZ and MN conceptualized and designed the study, YQD drafted the initial manuscript, analyzed and interpreted the data and revised the manuscript for important intellectual content. JYS, HYM and JMS were involved in the data collection and analysis. MN and YGZ coordinated and supervised data collection, critically reviewed. All authors have read and approved the final manuscript.

\section{Acknowledgments}


Not applicable.

Author details

Department of orthopaedics, Chinese People's Liberation Army General Hospital, 28 Fuxing Road, Haidian District, Beijing, China

\section{References}

1. Tamura K, Takao M, Hamada H, Ando W, Sakai T, Sugano N: Femoral morphology asymmetry in hip dysplasia makes radiological leg length measurement inaccurate. The bone \& joint journal 2019, 101-b(3):297-302.

2. Xu H, Zhou Y, Liu Q, Tang Q, Yin J: Femoral morphologic differences in subtypes of high developmental dislocation of the hip. Clinical orthopaedics and related research 2010, 468(12):33713376.

3. Sugano N, Noble PC, Kamaric E, Salama JK, Ochi T, Tullos HS: The morphology of the femur in developmental dysplasia of the hip. The Journal of bone and joint surgery British volume 1998, 80(4):711-719.

4. Greber EM, Pelt CE, Gililland JM, Anderson MB, Erickson JA, Peters CL: Challenges in Total Hip Arthroplasty in the Setting of Developmental Dysplasia of the Hip. The Journal of arthroplasty 2017, 32(9s):S38-s44.

5. Zhou Y, Sun C, Wang Y: New Method Addressing the Problem of Using Ceramic-on-Ceramic Bearing in Too Small Acetabulum of High-Riding DDH Patients with THA. Seminars in Arthroplasty 2012, 23(4):226-231.

6. Albinana J, Morcuende JA, Delgado E, Weinstein SL: Radiologic pelvic asymmetry in unilateral latediagnosed developmental dysplasia of the hip. Journal of pediatric orthopedics 1995, 15(6):753-762.

7. Jia J, Zhang L, Zhao Q, Li L, Liu X: Does medial rotational deformity of the whole pelvis universally exist in unilateral DDH? Archives of orthopaedic and trauma surgery 2011, 131(10):1383-1388.

8. Bilgen OF, Salar N, Bilgen MS, Mutlu M, Kara GK, Gursel E: The Effect of Dislocation Type (Crowe Types I-IV) on Pelvic Development in Developmental Dysplasia of the Hip: A Radiologic Study of Anatomy. The Journal of arthroplasty 2015, 30(5):875-878.

9. Zhang Z, Luo D, Cheng H, Xiao K, Zhang H: Unexpected Long Lower Limb in Patients with Unilateral Hip Dislocation. The Journal of bone and joint surgery American volume 2018, 100(5):388-395.

10. Argenson JN, Ryembault E, Flecher X, Brassart N, Parratte S, Aubaniac JM: Three-dimensional anatomy of the hip in osteoarthritis after developmental dysplasia. The Journal of bone and joint surgery British volume 2005, 87(9):1192-1196.

11. Welton KL, Jesse MK, Kraeutler MJ, Garabekyan T, Mei-Dan O: The Anteroposterior Pelvic Radiograph: Acetabular and Femoral Measurements and Relation to Hip Pathologies. The Journal of bone and joint surgery American volume 2018, 100(1):76-85. 
12. Kjellberg M, Al-Amiry B, Englund E, Sjoden GO, Sayed-Noor AS: Measurement of leg length discrepancy after total hip arthroplasty. The reliability of a plain radiographic method compared to CT-scanogram. Skeletal Radiol 2012, 41(2):187-191.

13. Flecher $X$, Ollivier M, Argenson JN: Lower limb length and offset in total hip arthroplasty. Orthopaedics \& traumatology, surgery \& research : OTSR 2016, 102(1 Suppl):S9-20.

14. Ma HY, Zhou YG, Zheng C, Cao WZ, Wang S, Wu WM, Piao S, Du YQ: New classification of Crowe type IV developmental dysplasia of the hip. Zhongguo gu shang = China journal of orthopaedics and traumatology 2016, 29(2):119-124.

15. Kakaty DK, Fischer AF, Hosalkar HS, Siebenrock KA, Tannast M: The ischial spine sign: does pelvic tilt and rotation matter? Clinical orthopaedics and related research 2010, 468(3):769-774.

16. Tannast M, Pfannebecker P, Schwab JM, Albers CE, Siebenrock KA, Buchler L: Pelvic morphology differs in rotation and obliquity between developmental dysplasia of the hip and retroversion. Clinical orthopaedics and related research 2012, 470(12):3297-3305.

17. Xu J, Xu C, Mao Y, Zhang J, Li H, Zhu Z: Posterosuperior Placement of a Standard-Sized Cup at the True Acetabulum in Acetabular Reconstruction of Developmental Dysplasia of the Hip With High Dislocation. The Journal of arthroplasty 2016, 31(6):1233-1239.

18. Fujii M, Nakashima Y, Sato T, Akiyama M, Iwamoto Y: Pelvic deformity influences acetabular version and coverage in hip dysplasia. Clinical orthopaedics and related research 2011, 469(6):1735-1742.

19. Li YM, Li JH, Li B, Wang JX, Chen YS: The radiological research for pelvis asymmetry of unilateral developmental dysplasia of the hip in adult. Saudi medical journal 2016, 37(12):1344-1349.

20. Meermans G, Malik A, Witt J, Haddad F: Preoperative radiographic assessment of limb-length discrepancy in total hip arthroplasty. Clinical orthopaedics and related research 2011, 469(6):16771682.

21. Woolson ST, Hartford JM, Sawyer A: Results of a method of leg-length equalization for patients undergoing primary total hip replacement. The Journal of arthroplasty 1999, 14(2):159-164.

22. Erkula G, Celikbas E, Kilic BA, Demirkan F, Kiter AE: The acetabular teardrop and ultrasonography of the hip. J Pediatr Orthop B 2004, 13(1):15-20.

23. Albinana J, Morcuende JA, Weinstein SL: The teardrop in congenital dislocation of the hip diagnosed late. A quantitative study. The Journal of bone and joint surgery American volume 1996, 78(7):10481055.

24. Tonnis D: Normal values of the hip joint for the evaluation of X-rays in children and adults. Clinical orthopaedics and related research 1976(119):39-47.

25. Smith JT, Matan A, Coleman SS, Stevens PM, Scott SM: The predictive value of the development of the acetabular teardrop figure in developmental dysplasia of the hip. Journal of pediatric orthopedics 1997, 17(2):165-169.

\section{Tables}


Table 1. Patient demographic characteristics

\begin{tabular}{lccc}
\hline Variable & Type IVA & Type IVB & P values \\
\hline Patients (n) & 48 & 41 & \\
Gender (n) & 3 & 2 & 0.779 \\
Male & 45 & 39 & \\
Female & $38.6 \pm 12.0$ & $38.5 \pm 9.8$ & 0.983 \\
Age $(\mathrm{yrs})^{*}$ & $158.3 \pm 6.3$ & $158.6 \pm 6.6$ & 0.685 \\
Height $(\mathrm{cm})^{*}$ & $56.6 \pm 9.7$ & $58.0 \pm 10.5$ & 0.527 \\
Weight $(\mathrm{kg})^{*}$ & $22.5 \pm 2.9$ & $23.0 \pm 3.5$ & 0.593 \\
BMI $\left(\mathrm{kg} / \mathrm{m}^{2}\right)^{*}$ & 34 & 19 & 0.019 \\
Affected side (n) & 14 & 22 & \\
Right & & & \\
\hline Left & & & \\
\hline
\end{tabular}

Values are expressed as the mean and standard deviation. BMI: body mass index.

\section{Figures}


Table 2. Descriptions and reliability/reproducibility of the eight measurement parameters (Fig. 1).

Parameters Description

Interobserver

ICC

Intraobserver

ICC

\begin{tabular}{|c|c|c|c|c|c|}
\hline $\begin{array}{l}\text { Height of } \\
\text { ilium area } \\
\text { (Line m) }\end{array}$ & $\begin{array}{l}\text { A vertical line (Line a) is drawn } \\
\text { from iliac crest vertex to the } \\
\text { bisector of the pelvis (Line k), and a } \\
\text { vertical line (Line b) is drawn from } \\
\text { inferior sacroiliac articulation to the } \\
\text { Line k. The height of ilium area was } \\
\text { the vertical distance between the } \\
\text { Line a and Line b. }\end{array}$ & $\begin{array}{l}0.95 \\
0.97)\end{array}$ & $(0.91-$ & $\begin{array}{l}0.96 \\
0.98)\end{array}$ & $(0.94-$ \\
\hline $\begin{array}{l}\text { Height of } \\
\text { acetabulum } \\
\text { area (Line } \\
\text { n) }\end{array}$ & $\begin{array}{l}\text { A vertical line (Line c) is drawn } \\
\text { from teardrop to the Line k. The } \\
\text { height of acetabulum area was the } \\
\text { vertical distance between the Line b } \\
\text { and Line c. }\end{array}$ & $\begin{array}{l}0.92 \\
0.95)\end{array}$ & (0.89- & $\begin{array}{l}0.93 \\
0.95)\end{array}$ & $(0.90-$ \\
\hline $\begin{array}{l}\text { Height of } \\
\text { ischium } \\
\text { area (Line } \\
\text { o) }\end{array}$ & $\begin{array}{l}\text { A vertical line (Line d) is drawn } \\
\text { from ischial tuberosity to the Line k. } \\
\text { The height of acetabulum area was } \\
\text { the vertical distance between the } \\
\text { Line c and Line d. }\end{array}$ & $\begin{array}{l}0.92 \\
0.94)\end{array}$ & (0.89- & $\begin{array}{l}0.96 \\
0.97)\end{array}$ & (0.94- \\
\hline $\begin{array}{l}\text { Width of } \\
\text { ilium (Line } \\
\text { p) }\end{array}$ & $\begin{array}{l}\text { A line (Line e) is passed through the } \\
\text { inferior sacroiliac articulation, } \\
\text { parallel to the Line } \mathrm{k} \text {. The other line } \\
\text { (Line f) is passed through the lateral } \\
\text { margin of the ilium, parallel to the } \\
\text { Line k. The distance between the } \\
\text { two lines is defined as width of } \\
\text { ilium. }\end{array}$ & $\begin{array}{l}0.98 \\
0.99)\end{array}$ & (0.98- & $\begin{array}{l}0.98 \\
0.99)\end{array}$ & (0.97- \\
\hline $\begin{array}{l}\text { The } \\
\text { discrepancy } \\
\text { of iliac } \\
\text { crest (Line } \\
\text { q) }\end{array}$ & $\begin{array}{l}\text { The discrepancy of iliac crest (Line } \\
\text { g) is the distance between two sides } \\
\text { of Line a. }\end{array}$ & $\begin{array}{l}0.93 \\
0.95)\end{array}$ & (0.90- & $\begin{array}{l}0.91 \\
0.94)\end{array}$ & (0.87- \\
\hline $\begin{array}{l}\text { The } \\
\text { discrepancy } \\
\text { of inferior } \\
\text { sacroiliac } \\
\text { articulation } \\
\text { (Line h) }\end{array}$ & $\begin{array}{l}\text { The discrepancy of inferior } \\
\text { sacroiliac articulation (Line h) is the } \\
\text { distance between two sides of Line } \\
\text { b. }\end{array}$ & $\begin{array}{l}0.94 \\
0.96)\end{array}$ & (0.88- & $\begin{array}{l}0.95 \\
0.97)\end{array}$ & (0.92- \\
\hline $\begin{array}{l}\text { The } \\
\text { discrepancy } \\
\text { of teardrop } \\
\text { (Line i) }\end{array}$ & $\begin{array}{l}\text { The discrepancy of teardrop (Line i) } \\
\text { is the distance between two sides of } \\
\text { Line c. }\end{array}$ & $\begin{array}{l}0.91 \\
0.94)\end{array}$ & (0.85- & $\begin{array}{l}0.91 \\
0.92)\end{array}$ & (0.89- \\
\hline $\begin{array}{l}\text { The } \\
\text { discrepancy } \\
\text { of ischial } \\
\text { tuberosity } \\
\text { (Line j) }\end{array}$ & $\begin{array}{l}\text { The discrepancy of ischial tuberosity } \\
\text { (Line j) is the distance between two } \\
\text { sides of Line d. }\end{array}$ & $\begin{array}{l}0.90 \\
0.92)\end{array}$ & (0.86- & $\begin{array}{l}0.91 \\
0.92)\end{array}$ & (0.91- \\
\hline
\end{tabular}

The values are expressed as mean, with 95\% confidence interval. ICC: intraclass correlation coefficient. 


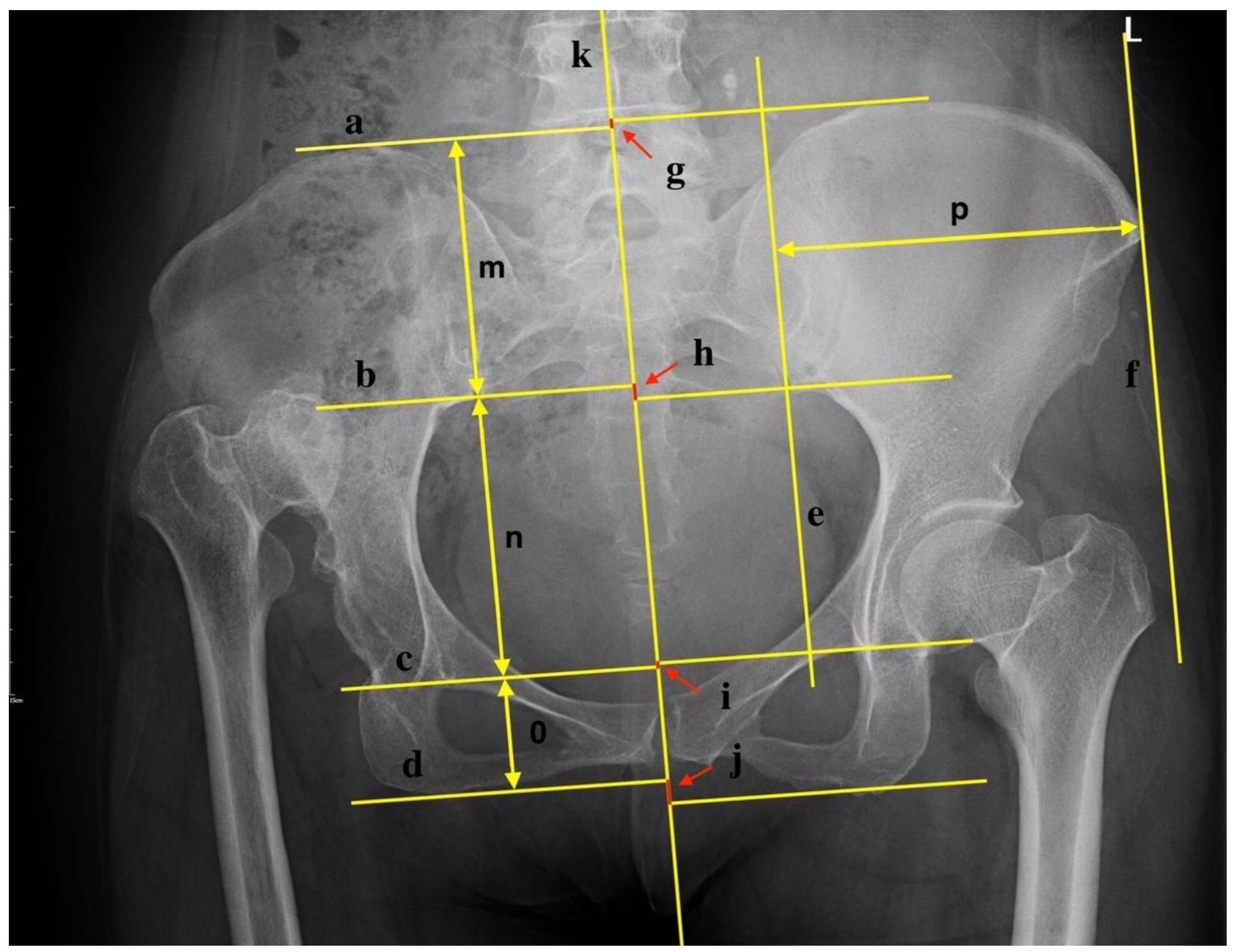

\section{Figure 1}

Measurement parameters determined on AP pelvic radiographs: $(m)$ Height of ilium area, (n) Height of acetabulum area, (0) Height of ischium area, (p) Width of ilium, (g) The discrepancy of iliac crest, (h) The discrepancy of inferior sacroiliac articulation, (i) The discrepancy of teardrop, (j) The discrepancy of ischial tuberosity, see Table 2. 


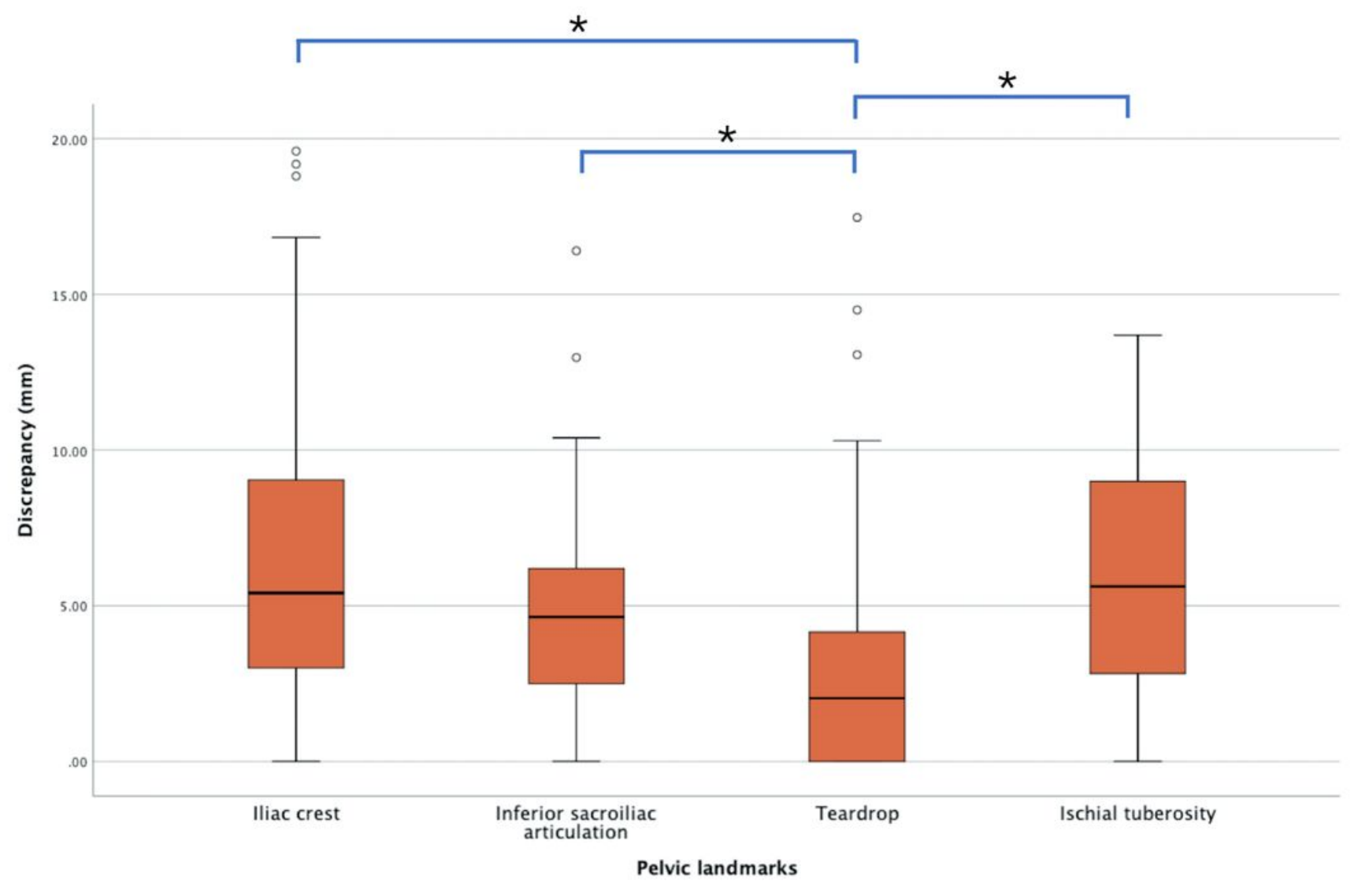

Figure 2

Box plots showing the discrepancies of bilateral iliac crest, inferior sacroiliac articulation, teardrop and ischial tuberosity on the line of the bisector of the pelvis. A statistically significant difference $(P<0.05)$ in teardrop versus iliac crest, teardrop versus inferior sacroiliac articulation, teardrop versus ischial tuberosity. 